\title{
Assimilation capabilities of the IRI-Plas model
}

\author{
Olga A. Maltseva* \\ * Institute for Physics, Southern Federal University \\ 344090, Rostov-on-Don, Russia \\ e-mail:mal@ip.rsu.ru
}

\begin{abstract}
In studies of the ionosphere, empirical models play no less a role than experimental data. The most common is the IRI model [1], which describes well the behavior of such parameters as the critical frequency foF2 and the maximum height hmF2. The model allows assimilation of these parameters while adapting to the data of the current diagnostics. But in connection with the appearance of navigation satellites and the measurement of the total electronic content TEC, the IRI-Plas model appeared, which allows the assimilation of the TEC [2]. This can provide great advantages in studying the behavior of the ionosphere, in particular, the parameter foF2, during disturbances along the meridians. One of the possible approaches is assimilation of the IRI-Plas model to the latitudinal dependence of TEC. To test this approach, it is proposed to use a network of ionosondes along the meridian. The ionosonde data at each point is used to calculate the equivalent slab thickness $\tau$ of the ionosphere, and from its values at these points, a polynomial latitudinal dependence of $\tau$ is constructed. Using this dependence and the observational TEC values along the meridian, the foF2(rec) values calculated using $\tau$ and foF2(Plas) obtained by assimilation of the TEC into the IRI-Plas model are determined and compared. The effectiveness of this procedure is illustrated by the example of the $15^{\circ}$ E meridian and the known disturbances of March 2012 . Five ionosondes are located along this meridian: Longyearbyen, Tromso, Juliusruh, Pruhonice, Rome. According to the data of these ionosondes, it was shown that the frequencies foF2(rec) correspond to the observational values of foF2(obs) with absolute deviations $\mid$ foF2(rec) $\mid$ in the range of $0.3-0.4 \mathrm{MHz}$. To determine the TEC, JPL global maps are used in the range of $30^{\circ} \mathrm{N}-80^{\circ} \mathrm{N}$ with a step of $2.5^{\circ}$. These values are substituted into the IRI-Plas model and the foF2(Plas) values are calculated. To assess the efficiency of assimilation, two coefficients are introduced: $\eta(\mathrm{TEC})=\mathrm{TEC}(\mathrm{obs}) / \mathrm{TEC}(\mathrm{Plas})$ and $\eta($ foF 2$)=$ foF2(rec)/foF2(Plas). In the latitudinal course, the relative error can be defined as (1$1 / \eta)^{*} 100 \%$. The absolute TEC deviations were in the range of $0.5-1$ TECU, the relative deviations were in the 3.5-6\% range and increased with increasing latitude. For foF2, an additional comparison with the IRI model is given. IRI-Plas always gives better results than IRI. For latitudes exceeding $45^{\circ} \mathrm{N}, \mid \Delta$ foF2(Plas) $\mid$ varies from $0.6 \mathrm{MHz}$ to $0.2 \mathrm{MHz}$ with increasing latitude, for IRI it is almost constant $(\sim 0.5 \mathrm{MHz})$, the relative deviations for foF2(Plas) decrease from $10 \%$ to $5 \%$, for IRI, they are almost constant around $10 \%$. A noticeable increase is observed at low latitudes. Despite the fact that the difference between the results of IRI-Plas and IRI is only 1.5-2 times, it should be noted that IRI-Plas reflects the nature of disturbances much better. The constructed successive latitudinal dependences of foF2(Plas) show day-to-day variations that are in good agreement with the behavior of foF2 in individual latitudinal zones noted in separate papers. Thus, this approach can be used to study variations in foF 2 during disturbances on the meridians not provided by ionosondes.
\end{abstract}

\section{REFERENCES}

[1] D. Bilitza, D. Altadill, V. Truhlik, et al., "IRI 2016: From ionospheric climate to real-time weather predictions," Space Weather, Vol. 15, pp. 418 - 429, (2017).

[2] T.L. Gulyaeva, X. Huang, and B.W. Reinisch, "Plasmaspheric extension of topside electron density profiles", Adv. Space Res., Vol. 29(6), pp. 825-831. (2002). 